\title{
Management of Temporomandibular Joint Disorders by Arthroscopic Lavage
}

\author{
Rab MA ${ }^{1}$, Chowdhury $\mathrm{GM}^{2}$, Al-Mehedi $\mathrm{A}^{3}$, Sindhi $\mathrm{Q}^{4}$, Kabir $\mathrm{MI}^{5}$, Sharmin $\mathrm{S}^{6}$
}

\begin{abstract}
Introduction: Temporomandibular joint disorders (TMDs) are among the most common disorders in the maxillofacial region which usually present with joint pain, decreased jaw mobility, joint clicking, headache, tinnitus, neck pain etc. There are different modalities of treatment used for the TMDs. These include some conservative and surgical procedures. The main aim is to improve the jaw function and reduction of joint pain. Arthroscopy is a modern diagnostic and effective therapeutic procedure for the TMDs.
\end{abstract}

Objective: To assess the outcome of arthroscopic lavage of Temporomandibular Joint (TMJ) in terms of improvement of jaw function and joint pain in patients suffering from TMDs.

Materials and Methods: This prospective study was carried out at the department of Oral and Maxillofacial Surgery, Military Dental Centre, Combined Military Hospital of Dhaka Cantonment from 01 October 2014 to 31 March 2016. Patients were selected consecutively having TMDs and unresponsive to conservative nonsurgical therapy. Total thirty patients of TMDs were selected for study and all the patients were treated with arthroscopic lavage. Objective data were collected including, inter incisal opening, lateral excursion, deviation on opening, tenderness on palpation. A questionnaire in the form of visual analogue scale relating to pain, joint clicking and Jaw mobility were completed by each patient at 1 week, 1 month and 6 months post operatively.

Results: Among thirty patients, 10 patients were male and 20 patients were female. Mean age of the patient was $42.6 \pm 5.79$ years. Maximum patients were in the age group of $41-45$ years. There was statistically significant improvement in maximum incisal opening and pain score. There was also reduction in Joint clicking and tenderness in maximum patients.

Conclusion: The findings of the present study suggested potential utility of arthroscopic lavage in the management of TMDs.

Key-words: Temporomandibular Joint (TMJ), Temporomandibular disorders (TMDs).

\section{Introduction}

Temporomandibular disorders (TMDs) represent a wide range of functional changes and pathological conditions affecting the temporomandibular joint (TMJ), masticatory muscles, and other components of the oromaxillofacial region. In recent years, TMD has become a frequent cause for seeking medical assistance. The number of patients with TMDs is increasing, probably due to psychological tension in modern society ${ }^{1}$. According to well accepted psychophysiological concepts, occlusal problems and emotional stress are the most serious aetiological factors ${ }^{2,3}$. However, the causes of TMD are far more complex. A comprehensive understanding requires consideration of the whole masticatory apparatus and the intra-articular situation ${ }^{1}$.

TMD is seen most commonly in people between the ages of 20 and 40 years and occurs more often in women than in men ${ }^{4-6}$. Signs and symptoms of TMDs may be pain, impaired jaw function, malocclusion, deviation or deflection, limited range of motion, joint noise and locking. Headache, tinnitus, visual changes, and other neurologic complaints may also accompany $\mathrm{TMDS}^{7}$. TMJ dysfunction is a therapeutic challenge in the oral and

1. Brig Gen Md Abdur Rab, BDS, FCPS, OMF Surgeon and Commandant, Military Dental Centre, Jessore Cantonment 2. Maj Gen Golam Mohiuddin Chowdhury, BDS, FCPS, Consultant Dental Surgeon General, Bangladesh Armed Forces, Dte Gen Med Svcs, Dhaka Cantonment 3. Lt Col Abdullah AI-Mehedi, BDS, FCPS, Military Dental Centre, Dhaka Cantonment 4. Lt Col Quazi Sindhi, BDS, FCPS, Military Dental Centre, Dhaka Cantonment 5. Maj Mohammad Iqbal Kabir, BDS, MCPS, DOMFS, FCPS, Military Dental Centre, Chittagong Cantonment 6. Dr Shaila Sharmin, BDS, MS, Assistant Professor and Head, MH Samorita Medical College and Dental Unit, Tejgaon, Dhaka. 
maxillofacial clinic. Although TMJ pain and dysfunction can be caused by many different aetiological factors, the role played by inflammation as an underlying mechanism of pain and dysfunction of the TMJ has played a major role. TMD patients having pain and tenderness for prolonged period of time will show signs of inflammation biochemically as well as radiographically ${ }^{8}$.

The primary goal in the treatment of TMD is to ease pain or mandibular dysfunction ${ }^{9}$. The treatment options for patients with TMDs can range from conservative to surgical modalities ${ }^{10}$. Nonsurgical therapy may include counseling, anti-inflammatory medications, muscle relaxants, habit modification, splint therapy, exercise, physiotherapy (including moist heat, cold laser and ultrasonography) and a soft diet or some combination of these strategies. Surgical therapy ranges from minimally invasive procedures such as arthroscopy to a variety of open-joint procedures ${ }^{11,12}$.

Arthroscopy has the distinct advantage of allowing direct visualization of the TMJ and fulfills not only a therapeutic role but also a diagnostic one ${ }^{13}$. It also allows for lysis and lavage procedures under direct vision, as well as guided steroid injections ${ }^{14,15}$. Lysis and lavage of the TMJ were first done using arthroscopy by Ohnishi ${ }^{16}$.

Mechanical lysis of adhesions and lavage of the TMJ was often successful in treating various internal derangements ${ }^{17}$. Lavage of the upper joint compartment forces the flexible disc apart from the fossa, washes away degraded particles and inflammatory components and decreases the intra-articular pressure whenever the joint is inflamed. Arthroscopic lavage has been reported to reduce joint pain, improve joint function, and reduce clicking. It is most commonly used to treat patients with anterior disc displacement without reduction (closed lock) and disc adhesion. It is also used as a palliative treatment for acute episodes of degenerative or rheumatoid arthritis ${ }^{17,18}$

\section{Materials and Methods}

This prospective study was carried out at the department of Oral and Maxillofacial Surgery, Military Dental Centre, Combined Military Hospital, Dhaka Cantonment from 01 October 2014 to 31 March 2016. Patients were selected consecutively having TMD and unresponsive to conservative nonsurgical therapy. Total 30 patients were included in the study on the basis of some inclusion and exclusion criteria.
The patients with recent history of pain, limited mouth opening, restricted lateral movement and patients unresponsive to nonsurgical treatment were included in the study. Patients who had masticatory muscle diseases, osteoarthritis, pregnancy, lactation, previous history of arthroscopy and arthrocentesis were excluded from the study. Patients with a history of bony or fibrous adhesion, condylar fractures and with psychological problems were also excluded from the study. Informed consent was taken from all the patients.

The clinical examination was done including the evaluation of the maximal mouth opening measured by the distance between the incisal edges of the upper and lower incisors with the help of a caliper. Determination of the 'range of the lateral and protrusive mandibular movement' measured by the distance between the upper and lower midline on lateral and forward movements by using a caliper.

Pain level and location were determined by the patients self assessment using Visual analogue scale $(0=$ no pain and $10=$ severe pain $)^{19}$. Joint clicking was evaluated by joint score $(0=$ no sound heard even by stethoscope, 1=mild sound heard just by stethoscope, 2=moderate click that can be felt by palpation and $3=$ severe sound audible by the patient or others) ${ }^{20}$. After thorough TMJ evaluation and clinical examination, orthopantamogram and cone beam CT scan were done in all the patients. Routine investigations were also done. The diagnosis of TMD was made on the basis of history, clinical and radiological examination as per the norms laid down by Kaplan ${ }^{21}$.

After proper preparation of the target site, external auditory meatus was blocked with cotton soaked in normal saline. Two points of insertion were marked over the skin. A line was drawn from the middle of the tragus to the outer canthus. The posterior entrance point was located along the cantho-tragal line, $10 \mathrm{~mm}$ from the middle of the tragus and $2 \mathrm{~mm}$ below the line. The exit point was located $10 \mathrm{~mm}$ further along the line and $10 \mathrm{~mm}$ below it. After a small stab incision a sharp obturator followed by a dull obturator was inserted in the upper joint cavity from posterior entry point and another sheath was inserted at anterior exit point. With the visualization of joint cavity by arthroscopy machine lactated Ringer's irrigation solution was injected through posterior entry point into the joint space to distend the capsule and it was coming out through anterior exit point (Fig-1 and Fig-2). 

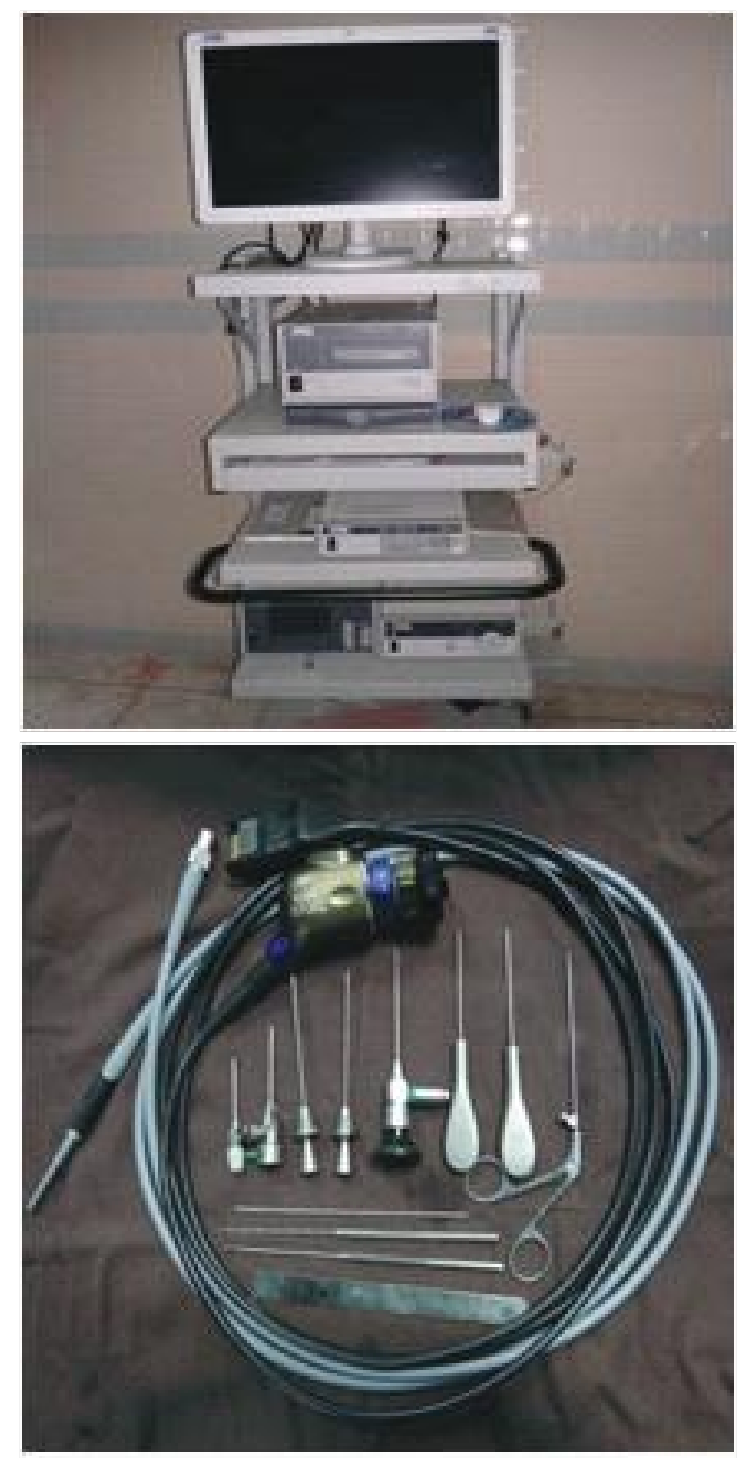

Fig-1: TMJ Arthroscopy Machine and Accessories.

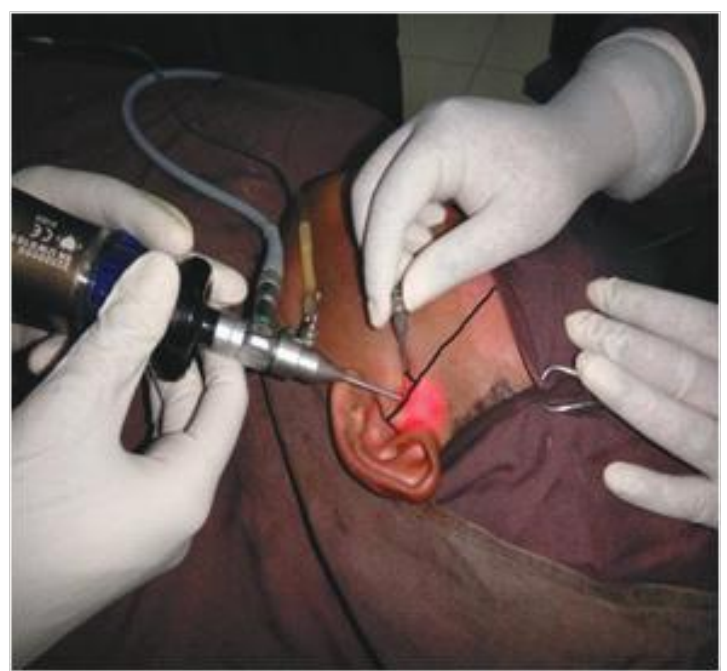

Fig-2: Arthroscopic lavage with double puncture technique.
Of 30 patients, 18 patients were treated with general anaesthesia and 12 patients with local anaesthesia as well as intravenous sedation. Depending on the symptom, bilateral TMJ lavage was done in 20 patients and unilateral lavage was done in 10 patients.

\section{Results}

The age of the patients ranged from 31 to 55 years with the mean age of $42.6 \pm 5.79$ years. The mean age of the male patients was $42.4 \pm 5.97$ years and that of female patients was $42.7 \pm 5.85$ years. There was no statistically significant difference between age of male and female patients ( $p>0.05)$. Maximum patients $(40 \%)$ were in the age group of $41-45$ years (table-I), followed by age group 36-40 years and $41-45$ years (20\% in each group).

Table-I: Frequency distribution of the patients as per age and sex of patients $(n=30)$

\begin{tabular}{|c|c|c|c|c|}
\hline \multirow{2}{*}{$\begin{array}{l}\text { Age group } \\
\text { in years }\end{array}$} & \multicolumn{2}{|r|}{ Sex } & \multirow{2}{*}{$\begin{array}{l}\text { Total } \\
\text { f (\%) }\end{array}$} & \multirow{2}{*}{$\begin{array}{l}\text { P (between } \\
\text { sex) }\end{array}$} \\
\hline & Male $(n=10) f(\%)$ & Female $(n=20) f(\%)$ & & \\
\hline $31-35$ & $1(10)$ & $2(10)$ & $03(10)$ & \multirow{7}{*}{$0.896^{*}$} \\
\hline $36-40$ & $2(20)$ & $4(20)$ & $06(20)$ & \\
\hline $41-45$ & $4(40)$ & $8(40)$ & $12(40)$ & \\
\hline $46-50$ & $2(20)$ & $4(20)$ & $06(20)$ & \\
\hline Above 50 & $1(10)$ & $2(10)$ & $03(10)$ & \\
\hline Total & $10(33.3)$ & $20(66.7)$ & $30(100)$ & \\
\hline Mean?SD & $42.4 \pm 5.97$ & $42.7 \pm 5.85$ & $42.6 \pm 5.79$ & \\
\hline
\end{tabular}

*Difference between the mean of two sexes is not significant

Table-II: Changes of maximal mouth opening after arthroscopic lavage $(n=30)$

\begin{tabular}{|l|c|c|c|}
\hline \multirow{2}{*}{\multicolumn{1}{|c|}{ Group }} & \multicolumn{3}{|c|}{ Maximal mouth opening (mm) } \\
\cline { 2 - 4 } & Range & Mean & SD \\
\hline Before the procedure & $20-40$ & 29.87 & 6.263 \\
\hline 1 week after & $24-45$ & 35.43 & 5.888 \\
\hline 1 month after & $33-47$ & 40.43 & 4.281 \\
\hline 6 month after & $41-49$ & 44.97 & 2.942 \\
\hline Comparison & \multicolumn{1}{|c|}{ T/F } & P \\
\hline Before Vs after 1 week & 3.547 & \multicolumn{2}{c|}{$0.001^{*}$} \\
\hline Before Vs after 1 month & 7.629 & \multicolumn{2}{c|}{$0.000^{*}$} \\
\hline Before Vs after 6 months & 11.953 & \multicolumn{2}{|c|}{$0.000^{*}$} \\
\hline ANOVA & 50.273 & $0.000^{*}$ \\
\hline
\end{tabular}

* Differences are highly significant

Before starting intervention, the maximal mouth opening was $29.87 \pm 6.26 \mathrm{~mm}$ with a range of $20-40$ $\mathrm{mm}$ which was increased to $44.97 \pm 2.94 \mathrm{~mm}(p<0.001)$ with a range of $41-49 \mathrm{~mm}$ after 6 month of arthroscopic lavage (Table-II). 
All the patients had restricted lateral movement of mandible before starting the procedure and the mean lateral movement was $6.47 \pm 1.06 \mathrm{~mm}$ on the right side and $6.50 \pm 0.98 \mathrm{~mm}$ on the left side. Six months after the arthroscopic lavage, mean lateral movement was increased to $9.50 \pm 0.77$ on the right side and $9.37 \pm 0.78 \mathrm{~mm}$ on the left side (Table-III). The differences are statistically highly significant $(p<0.001)$.

Table-III: Change of lateral mandibular movements after arthroscopic lavage $(n=30)$

\begin{tabular}{|c|c|c|c|c|c|c|}
\hline \multirow{3}{*}{ Group } & \multicolumn{6}{|c|}{ Lateral mandibular movements } \\
\hline & & \multicolumn{3}{|c|}{ Left } \\
\hline & \multicolumn{2}{|r|}{$\begin{array}{l}\text { Right } \\
\text { Mean }\end{array}$} & SD & Range & Mean & SD \\
\hline e the procedure & $5-8$ & 6.47 & 1.066 & $5-8$ & 6.50 & 0.983 \\
\hline $\mathrm{k}$ after & $6-9$ & 7.57 & 0.796 & $6-9$ & 7.40 & 0.747 \\
\hline nth after & $7-10$ & 8.20 & 1.710 & $7-10$ & 8.35 & 0.734 \\
\hline nth after & $8-11$ & 9.50 & 0.766 & $8-11$ & 9.37 & 0.776 \\
\hline \multirow{2}{*}{ parison } & \multicolumn{3}{|c|}{ Right } & \multicolumn{3}{|c|}{ Left } \\
\hline & \multicolumn{2}{|c|}{$\mathrm{T} / \mathrm{F}$} & p & $\mathrm{T} / \mathrm{F}$ & \multicolumn{2}{|r|}{ p } \\
\hline e Vs after 1 week & \multicolumn{2}{|c|}{3.993} & $0.000^{*}$ & 4.528 & \multicolumn{2}{|r|}{$0.000^{*}$} \\
\hline e Vs after 1 month & \multicolumn{2}{|c|}{8.275} & $0.000^{*}$ & 4.711 & \multicolumn{2}{|r|}{$0.000^{*}$} \\
\hline e Vs after 6 months & \multicolumn{2}{|c|}{12.54} & $0.000^{*}$ & 12.657 & \multicolumn{2}{|r|}{$0.000^{*}$} \\
\hline VA & \multicolumn{2}{|c|}{36.445} & $0.000^{*}$ & 68.543 & \multicolumn{2}{|r|}{$0.000^{*}$} \\
\hline
\end{tabular}

Table-IV: Change in degree of pain after arthroscopic lavage $(n=30)$

\begin{tabular}{l|c|c|c|}
\hline \multirow{2}{*}{ Group } & \multicolumn{3}{|c}{ Degree of pain (0-10) } \\
\cline { 2 - 4 } & Range & Mean & SD \\
\hline re the procedure & $6-10$ & 7.73 & 1.413 \\
\hline ek after & $5-9$ & 6.27 & 1.202 \\
\hline nth after & $2-6$ & 3.87 & 1.042 \\
\hline nth after & $1-4$ & 1.83 & 0.986 \\
\hline parison & T / F & P \\
\hline re Vs after 1 week & 4.332 & $0.000^{*}$ \\
\hline re Vs after 1 month & 12.067 & $0.000^{*}$ \\
\hline re Vs after 6 months & 18.762 & $0.000^{*}$ \\
\hline VA & 148.235 & $0.000^{*}$ \\
\hline \multicolumn{2}{|c|}{ *Differences are highly significant }
\end{tabular}

The mean pain score among the studied patients was 7.73 in visual analogue scale before the procedure and was significantly reduced $(p<0.001)$ six months after arthroscopic lavage (Table-IV). The mean joint clicking score was $1.73 \pm 1.13$ with a range of $1-3$ before procedure and after six months of arthroscopic lavage the mean joint clicking score was reduced to $0.026 \pm 0.46$ (Table-V). The differences are statistically highly significant.
Table-V: Change in degree of joint clicking after arthroscopic lavage $(n=30)$

\begin{tabular}{|l|c|c|c|}
\hline \multirow{2}{*}{ Group } & \multicolumn{4}{|c|}{ Degree of Joint Clicking(0-3) } \\
\cline { 2 - 4 } & Range & Mean & SD \\
\hline Before the procedure & $1-3$ & 1.73 & 0.740 \\
\hline 1 week after & $0-3$ & 1.43 & 0.858 \\
\hline 1 month after & $0-2$ & 0.63 & 0.669 \\
\hline 6 month after & $0-1$ & 0.27 & 0.450 \\
\hline Comparison & T / F & P \\
\hline Before Vs after 1 week & 1.45 & 0.152 \\
\hline Before Vs after 1 month & 6.042 & $0.000^{*}$ \\
\hline Before Vs after 6 months & 9.280 & & $0.000^{*}$ \\
\hline ANOVA & 28.897 & $0.000^{*}$ \\
\hline \multicolumn{4}{|c|}{ * Differences are highly significant } \\
\hline
\end{tabular}

\section{Discussion}

Temporomandibular joint disorder (TMD) is one of the common but neglected condition in Bangladesh like most of the third world countries. It is one of the most challenging diseases of modern society, diagnostically, prognostically and in terms of treatment.

TMD most commonly occurs in age group between $20-50$ years $^{22,23}$. In this study most common age group observed was $41-45$ years $(40 \%)$, followed by the age group $36-40$ and $46-50$ (20\% in each group) and of the total patients the age ranged from 31 to 55 years. The mean age of the studied patients was $42.6 \pm 5.79$ years. In the present study male female frequency ratio was 1:2. Similar results were observed by many studies that TMDs occur more frequently in female than male ${ }^{24-27}$.

The role of arthroscopic lavage and accompanying process of arthrolysis has shown excellent success rates in treatment of TMDs. This process has shown to reduce pain and improve joint mobility, sometimes even effective in relief of patients' suffering from advanced stages of degeneration and dysfunction ${ }^{17,28,29}$. In the present study it was revealed that there was significant improvement of joint pain and jaw mobility $(p<0.000)$. Arthroscopic lavage has been advocated as a potentially more effective than simple arthrocentesis, because the larger diameter portal used in lavage would enable more extensive removal of inflammatory mediators ${ }^{30}$.

Significant improvement can be achieved by arthroscopic lysis and lavage to treat those patients with painful clicking or popping to release intra-articular adhesions and anteriorly displaced non reducing discs and to confirm 
other diagnostic findings that could warrant surgical intervention $^{17,31}$. In the present study there was also significant improvement of joint function and also significant reduction of joint pain and clicking after arthroscopic lavage $(p<0.001)$.

\section{Conclusion}

TMDs are frequent and wide spread in general population. The patients with TMD need surgical intervention only when nonsurgical therapy fails. Arthroscopic lavage is a minimally invasive surgical procedure for the treatment of TMDs. Clinical success of arthroscopy is based on several factors. Lysis and lavage remove intra articular inflammatory and pain mediators. The release of fibrillations and adherences as well as improvement in discal mobility allows to distribute the functional stresses on the articular tissues and adverse loading on the joints is decreased. The results of current study revealed that there was significant reduction in TMJ pain and joint clicking. There was also significant increase in maximal mouth opening, lateral movements after a period of 6 months follow up and suggested potential utility of arthroscopic lavage in the management of TMDs. Further research involving a larger sample and a longer follow up period is suggested along with extensive work involving specificity of technique and more knowledge on the aetiology of the TMDs.

\section{Acknowledgement}

We are grateful to Brig Gen Md Ahsan Habib, Professor and Head, Department of Anatomy, AFMC for his sincere help in writing the article, especially in statistical aspect and reporting the result part.

\section{References}

1. De Riu G, Stimolo M, Meloni SM et al. Arthrocentesis and Temporomandibular Joint Disorders: Clinical and Radiological Results of a Prospective Study. International Journal of Dentistry 2013; 2013:790648 (Published online 2013 Nov 11).

2. Meloni SM, deRiu G, Pisano M et al. Computer assisted dental rehabilitation in free flaps reconstructed jaws: one year follow-up of a prospective clinical study. British Journal of Oral and Maxillofacial Surgery 2012; 50(8):726-31.

3. Meloni SM, deRiu G, Pisano M et al. Immediate versus delayed loading of single mandibular molars. One-year results from a randomised controlled trial. European Journal of Oral Implantology 2012; 5(4):345-53.
4. Detamore MS and Athanasiou KA. Structure and function of the temporomandibular joint disc: Implications for tissue engineering. J Oral Maxillofac Surg 2003; 61(4):494-506.

5. Ethunandan M. Temporomandibular joint arthrocentesisMore questions than answers? J Oral Maxillofac Surg 2006; 64:952-55.

6. Van Loon JP, de Bont LGM, Stegenga B et al. Groningen temporomandibular joint prosthesis: Development and first clinical application. Int J Oral Maxillofac Surg2002; 31(1):44-52.

7. Fletcher MC, Piecuch JF, Lieblich SE. Anatomy and patho- physiology of the temporomandibular joint. In Peterson's Principles of Oral and Maxillofacial Surgery, 2nd ed. Edited by Miloro M. Hamilton (Ontario): BC Decker 2004; 933-47.

8. Kopp S, Wenneberg B, Haraldson T et al. The shortterm effect of intra-articular injections of sodium hyaluronate and corticosteroid on temporomandibular joint pain and dysfunction. J Oral Maxillofac Surg 1985; 43:429-35.

9. Karlis V, Glickman R. Nonsurgical management of temporomandibular disorders. In: Miloro M, Ghali GE, Larsen PE. Peterson's principles of oral and maxillofacial surgery. 2nd ed. Hamilton: BC Decker 2004; 949-50.

10. Weinberg S, Kryshtalskyj B, Psutka D et al. Operative arthroscopy of the temporomandibular joint. Oral Health 1992; 82(5):11-4, 16, 18-20.

11. Sanders B. Management of internal derangements of the temporo-mandibular joint. Semin Orthod 1995; 1(4):244-57.

12. Ohnuki T, Fukuda M, Nakata A et al. Evaluation of the position, mobility, and morphology of the disc by MRI before and after four different treatments for temporomandibular joint disorders. Dento Maxillofac Radiol 2006; 35:103-9.

13. Murakami K, Ono T. Temporomandibular joint arthroscopy by inferolateral approach. Int J Oral Maxillofac Surg 1986; 15(4):410-7.

14. Rosenberg I, Goss AN. The outcome of arthroscopic treatment of temporomandibular joint arthropathy. Aust Dent J 1999; 44(2):106-11.

15. Israel HA. Part-I: The use of arthroscopic surgery for treatment of temporomandibular joint disorders. J Oral Maxillofac Surg 1999; 57(5):579-82.

16. Ohnishi M. Arthroscopy and arthroscopic surgery of the temporomandibular joint (TMJ). Rev Stomatol Chir Maxillofac 1990; 91:143-50. 
17. Tozoglua S, Al-Belasyb FA, Dolwick MF. A review of techniques of lysis and lavage of the TMJ. British Journal of Oral and Maxillofacial Surgery 2011; 49:302-9.

18. Dolwick MF. Temporomandibular joint surgery for internal derangement. Dent Clin North Am 2007; 51(1):195-208.

19. Merskey $H$. The perception and measurement of pain. $J$ Psychosom Res 1973 Nov; 17(4): 251-5.

20. Onder ME, Tuz HH, Kocyigit $\mathrm{D}$ et al. Long-term results of arthrocentesis in degenerative temporomandibular disorders. Pathol Oral Radiol Endod 2009; 107(1):e1-5.2009;107(1):e1-5.

21. Kaplan AS, Assael LA (Eds.) Temporomandibular disorders: diagnosis and treatment. Philadelphia: WB Saunders Company 1991; 663-79.

22. Solberg WK, Woo MW, Honston JB. Prevalence of mandibular dysfunction in young adults. J Am Dent Assoc 1979; 98:25-34.

23. Van der Bijl A. Psychogenic pain in dentistry. Compendium 1995; 16:46-54.

24. Levin SR, Mckinney MW. Validating the TMJ scale in national sample 1000 patients: Demographic and epidemiologic characteristics. J Orofac Pan 1994; 8:25-35.

25. Dimithroulis G. Temporomandibular disorders: A clinical update. Brit Med J 1998; 317:190-4.
26. Velly AM, Gornitsky M, Phlippe P. Contributing factors to chronic myofascial pain: A case-control study. Pain 2003; 104:491-9.

27. Bagis B, Ayaz EA, Turgut $S$ et al. Gender Difference in Prevalence of Signs and Symptoms of Temporoman-dibular Joint Disorders: A Retrospective Study on 243 Consecutive Patients. Int. J Med Sci 2012; 9(7):539-44.

28. González-García R, Rodríguez-Campo FJ. Arthroscopic lysis and lavage versus operative arthroscopy in the outcome of temporomandibular joint internal derangement: A comparative study based on Wilkes stages. J Oral Maxillofac Surg 2011; 69(10): 2513-24.

29. Leibur $E$, Jagur $O$, Müürsepp $P$ et al. Long-term evaluation of arthroscopic surgery with lysis and lavage of temporomandibular joint disorders. J Cranio-Maxillofac Surg 2010; 38(8): 615-20.

30. Hobeich JB, Salameh ZA, Ismail E et al. Arthroscopy versus arthrocentesis. A retrospective study of disc displacement management without reduction. Saudi Med J 2007; 28(10): $1541-4$.

31. Laskin DM, Greene CS, Hylander WL (eds). Temporamandibular disorders: An evidence-based approach to diagnosis and treatment. Chicago: Quintessence 2006:441-81. 